\title{
Corneal changes in Pseudoexfoliative eyes
}

\author{
Dr. Praveen V, Dr. Soumya H V \\ (Department of ophthalmology, JSS Medical College / JSS University, India)
}

\begin{abstract}
Aim: To look for corneal changes in Pseudoexfoliative eyes and its resulting clinical implication.

Methods: This prospective study carried out at JSS Hospital, Mysore consisted of 100 consecutive patients with Pseudoexfoliation who presented to the outpatient department between January 2015 to December 2015. A detailed Ocular evaluation was done. Corneal changes were noted. Central corneal thickness was measured using ultrasound pachymetry

Results: Male to Female ratio was nearly 3:2. 20 patients had pterygium in one or both eyes. The mean tear film break up time was 13.85 seconds. Mean Central corneal thickness in Pseudoexfoliation with glaucoma eyes $(502.7 \pm 12.82 \mu)$ was found to be lower when compared with Mean Central corneal thickness in Pseudoexfoliation without glaucoma eyes $(518.36 \pm 13.21 \mu)$ and this was found to be statistically significant.

Conclusion: Climatically induced changes like pterygium, climatic droplet keratopathy are seen in Pseudoexfoliative eyes probably due to common etiology. Evaluation of central corneal thickness is necessary in all patients with Pseudoexfoliative glaucoma due to thinner cornea and the risk of underestimation of intra ocular pressure.
\end{abstract}

Keywords: Central Corneal Thickness, Cornea, Pachymetry, Pseudoexfoliation with glaucoma, Pseudoexfoliation without glaucoma,

\section{Introduction}

Pseudoexfoliation syndrome may be defined as a discrete clinical entity characterized by the synthesis and deposition of fine white granular material, upon and within ocular and orbital tissues [1]. In the anterior segment of the eye, it is characterized by the deposition on the anterior lens capsule, ciliary body, zonules, pupillary margin of this iris, corneal endothelium, anterior vitreous and trabecular meshwork [2]. It is now considered the most common identifiable specific entity leading to the development of open angle glaucoma [3]. In routine clinical practice, intraocular pressure (IOP) is one of the most important parameters in the detection and monitoring of glaucoma. Goldmann applanation tonometry has become the international "gold standard" for IOP measurements [4]. The aim of the study is to look for corneal changes in Pseudoexfoliative eyes and its resulting clinical implication.

\section{Methods}

This prospective study was carried out at JSS Hospital, Mysore. The study population consisted of 100 consecutive patients with Pseudoexfoliation who presented to the outpatient department between January 2015 to December 2015.

The diagnosis of Pseudoexfoliation was made based on:

- Deposition of Pseudoexfoliation material on the pupillary margin.

- Deposition of Pseudoexfoliation material on the anterior capsule of lens.

Preferably Right eye of the patient was considered for the study. Left eye was considered if right eye was pseudophakic.

Patients were then subjected to following Ophthalmic evaluation:

- $\quad$ Recording of visual acuity for distance using Snellen's chart.

- $\quad$ Measurement of Tear film Break up time

- $\quad$ Conjunctiva was examined for the presence of pterygia. Cornea was examined for the presence of climatic droplet keratopathy and guttae. The endothelium was examined to look for the presence of Pseudoexfoliative material.

- $\quad$ Intraocular pressure was measured using Perkins hand held Applanation tonometer.

- Stereoscopic evaluation of the fundus and optic disc with indirect ophthalmoscope and 90-D lens was done 
depending upon the media clarity.

- $\quad$ Central corneal thickness was measured using ultrasound pachymetry.

Glaucomatous optic nerve damage was diagnosed based on a combination of one or more of the following features with Intra ocular pressure $\geq 21 \mathrm{~mm} \mathrm{hg}$ :

- Vertical cup-disc ratio of 0.5 or more (excluding physiological cups).

- Vertical cup-disc ratio asymmetry of 0.2 or more.

- Characteristic glaucomatous excavation of the neuroretinal rim

The Chi-square significant/Fisher Exact test has been carried out to find out significance of proportions between mean age of incidence of Pseudoexfoliation in males and females and also to find out significance of proportions between mean central corneal thickness in Pseudoexfoliation eyes with and without glaucoma.

\section{RESULTS}

In this prospective study there were 100 patients (eyes) with the evidence of Pseudoexfoliation. This included 24 eyes with glaucomatous changes and 76 eyes without glaucoma.

\subsection{Age and Gender distribution}

The age and gender distribution of the subjects enrolled in the study was as below (Table 1).

Table 1: Age and Gender distribution

\begin{tabular}{|c|c|c|c|}
\hline \multirow{2}{*}{ Age in years } & \multicolumn{2}{|c|}{ Sex } & \multirow{2}{*}{ Total } \\
\cline { 2 - 3 } & Female & Male & \\
\hline $\mathbf{4 0 - 5 0}$ & 2 & 2 & 4 \\
\hline $\mathbf{5 1 - 6 0}$ & 16 & 20 & 36 \\
\hline $\mathbf{6 1 - 7 0}$ & 21 & 26 & 47 \\
\hline$>71$ & 4 & 9 & 13 \\
\hline Total & 43 & 57 & 100 \\
\hline Mean age & $\mathbf{6 4} \pm \mathbf{7 . 8 3}$ & $\mathbf{6 0 . 1 8} \pm \mathbf{7 . 5 2}$ & $\mathbf{6 1 . 7 9} \pm 7.58$ \\
\hline
\end{tabular}

The mean age of the subjects in the study was 61.79 years. There was a linear increase in the number of Pseudoexfoliation patients with age upto the age of 70 years. Most of the patients were seen in the 61-70 years age group. There were more males with Pseudoexfoliation than females in the study (57 Males vs. 43 Females). The average age at which Pseudoexfoliation was seen was higher in females than in males i.e., females developed the disease at later age than males and this was statistically significant $(\mathrm{p}<0.05)$.

\subsection{Climatically induced corneal and conjunctival alterations}

20 patients with Pseudoexfoliation had pterygium in one or both eyes. This was more commonly seen in males (13 patients) and the incidence increased with the age (Table 2).

Table 2: Climatically induced corneal and conjunctival alterations

\begin{tabular}{|c|c|c|}
\hline \multicolumn{2}{|c|}{ Pterygium } & Total \\
\hline Present & Absent & \\
\hline $\mathbf{2 0}$ & 80 & 100 \\
\hline
\end{tabular}

\subsection{Tear film break up time (TBUT) in patients with Pseudoexfoliation}

Results of tear film break up time test are given below (Table 3).

Table 3: TBUT in patients with Pseudoexfoliation

\begin{tabular}{|c|c|}
\hline $\begin{array}{c}\text { TBUT in } \\
\text { seconds }\end{array}$ & No. of eyes \\
\hline $\mathbf{1 0}$ & 30 \\
\hline $\mathbf{1 1 - 1 5}$ & 41 \\
\hline $\mathbf{1 1 5}$ & 29 \\
\hline Total & 100 \\
\hline
\end{tabular}


30 eyes had abnormal tear film break up time of $\leq 10$ seconds and in the rest of eyes (70) it was normal. The mean tear film break up time was 13.85 seconds.

\subsection{Corneal alterations in Pseudoexfoliation}

Most common associated condition noticed in cornea was climatic droplet keratopathy which was seen in 18 eyes. The corneal guttae which may be the complication of Pseudoexfoliation syndrome was seen in 5 eyes. Pseudoexfoliative material on cornea was seen in 5 eyes (Table 4).

Table 4: Corneal alterations in Pseudoexfoliation

\begin{tabular}{|c|c|c|c|}
\hline Corneal alterations & $\begin{array}{c}\text { Climatic Droplet } \\
\text { Keratopathy }\end{array}$ & Guttae & $\begin{array}{c}\text { Pseudoexfoliative } \\
\text { material on cornea }\end{array}$ \\
\hline No. of eyes & 18 & 5 & 5 \\
\hline
\end{tabular}

\subsection{Central Corneal Thickness (CCT) in Pseudoexfoliative eyes}

Mean CCT in Pseudoexfoliative eyes $(514.28 \pm 20.8 \mu)$ as compared with CCT in age matched normal population $(511.4 \pm 33.5 \mu$ ) [Vijaya L et al., Central Corneal Thickness in Adult South Indians][5] was almost similar.

However, Mean CCT in Pseudoexfoliation with glaucoma eyes $(502.7 \pm 12.82 \mu)$ was found to be lower when compared with Mean CCT in Pseudoexfoliation without glaucoma eyes $(518.36 \pm 13.21 \mu)$ and this was found to be statistically significant $(\mathrm{p}<0.05)$ (Fig 1$)$.

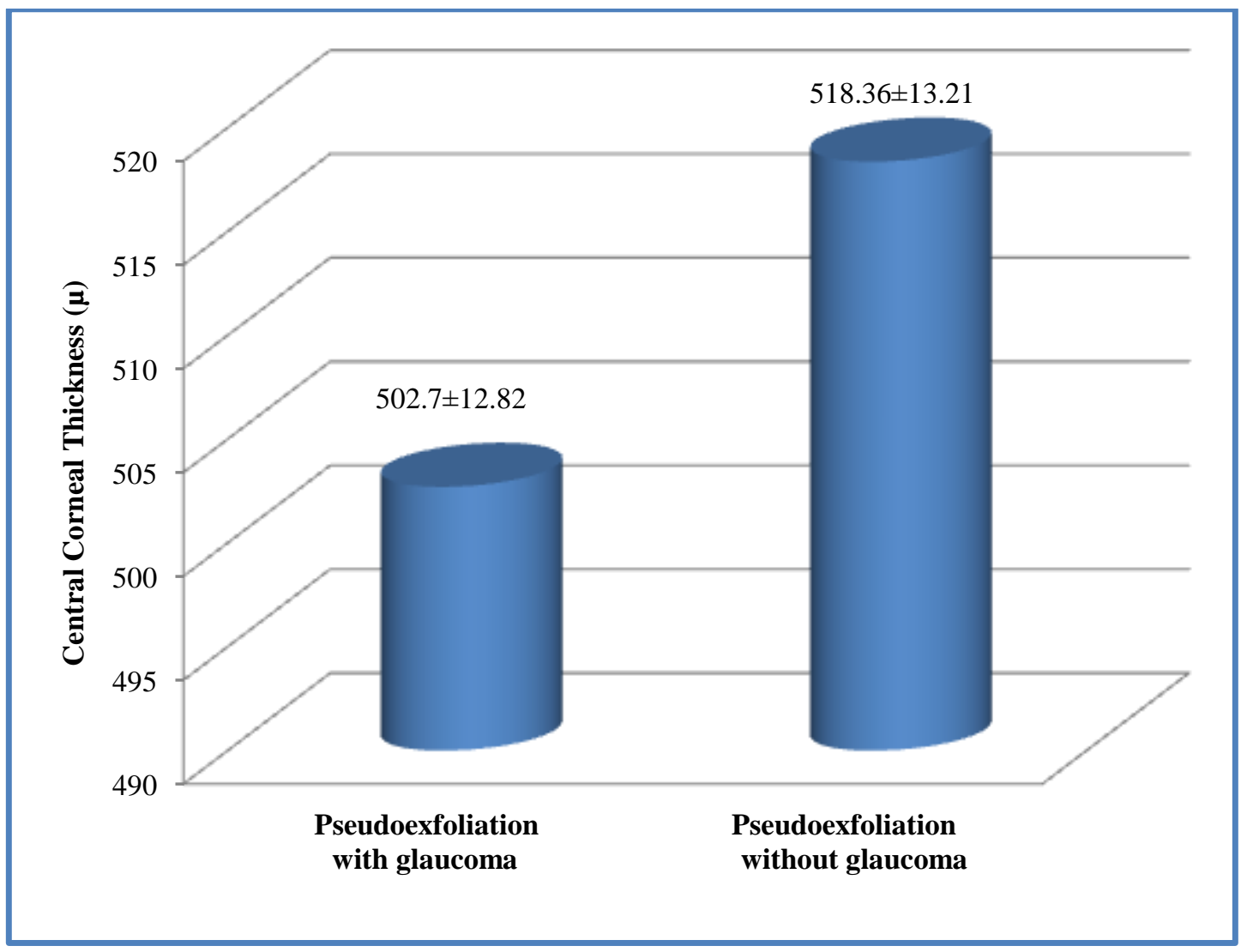

Fig 1: Central Corneal Thickness in Pseudoexfoliative eyes

\section{DISCUSSION}

\subsection{The association between exfoliation syndrome and disorders caused by climate}

In our study, we found that the incidence of pterygium and Pseudoexfoliation syndrome both peaked at 61-70 years of age and more males had pterygia and Pseudoexfoliation than females. This could point to a common etiology probably exposure to ultra violet radiation in the pathogenesis of both the conditions.

Forsius H, Forsman E et al however noted in their study that there was rapid increase in the incidence of Pseudoexfoliation with age, especially after 50 years. Whereas pterygium, climatic droplet keratopathy or 
pronounced pingecula reached its highest incidence after 60 years. They also found a higher incidence of pterygia, climatic droplet keratopathy and pingecula in males but did not find a corresponding higher incidence of Pseudoexfoliation syndrome in males in their study population [6].

Resnikoff S, Filliard G found six times as many cases of exfoliation syndrome in persons with climatic keratopathy than in people without [7]. Although we found climatic droplet keratopathy in 18 eyes, there was no comparison available because we did not have the control group.

\subsection{Tear film break up time in Pseudoexfoliation syndrome}

Our study had only a small proportion of patients with tear film break up time of $\leq 10$ secs. 70 patients had TBUT > 10 seconds. The mean TBUT was 13.85 seconds.

Kozobolis VP, Detorakis et al [8] found that the tear film break up times were significantly lower in Pseudoexfoliation syndrome. The average being 6.91 seconds, which was considerably lesser than that found in our study group. Probably the incidence of dry eye is more common in that region which could affect the test so significantly.

\subsection{Central Corneal Thickness (CCT)}

Mean CCT in Pseudoexfoliative eyes $(514.28 \pm 20.8 \mu)$ as compared with CCT in age matched normal population $(511.4 \pm 33.5 \mu$ ) [Vijaya L et al, Central Corneal Thickness in Adult South Indians] [5] was almost similar.

However, Mean CCT in Pseudoexfoliation with glaucoma eyes $(502.7 \pm 12.82 \mu)$ was found to be lower when compared with mean CCT in Pseudoexfoliation without glaucoma eyes $(518.36 \pm 13.21 \mu)$.

Georgios et al[9] in their study found CCT in Pseudoexfoliation with glaucoma eyes $(526 \mu)$ was significantly thinner compared to eyes with Pseudoexfoliation without glaucoma $(547.3 \mu)$.

\section{CONCLUSION}

Climatically induced changes like pterygium, climatic droplet keratopathy are seen in Pseudoexfoliative eyes probably due to common etiology. Evaluation of central corneal thickness is necessary in all patients with Pseudoexfoliative glaucoma due to thinner cornea and the risk of underestimation of intra ocular pressure.

\section{References}

[1]. Morrison, Green. Light microscopy of the exfoliation syndrome. Acta Ophthalmol Scand (Suppl).1988; 184: 5-27

[2]. Naumann GO, Schlotzer-Schrehardt U et al. Pseudoexfoliation syndrome for the comprehensive ophthalmologist: Intraocular and systemic manifestations. Ophthalmology. 1998; 105: 951-968.

[3]. Layden WE, Shaffer R. Exfoliation syndrome. Am J Ophthalmol 1974;78:835-41.

[4]. Doughty MJ, Zaman ML. Human corneal thickness and its impact on intraocular pressure measures: a review and metaanalysis approach. Surv Ophthalmol. 2000;44:367-408.

[5]. Vijaya L et al. Central corneal thickness in adult south Indians. Chennai Glaucoma Study. 2010 Jan; 30-35.

[6]. Forsius H, Forsman E et al. Exfoliation syndrome: Frequency, gender distribution and association with climatically induced alterations of the cornea and conjunctiva Acta Ophthalmologica Scandinavica 2002 Oct; 80(5): 465.

[7]. Resnikoff S, Filliard G. Climatic droplet keratopathy, exfoliation syndrome and cataract. Br J Ophthalmol. 1991; 75: 734-6.

[8]. Kozobolis VP, Detorakis et al. Evaluation of tear secretion and tear film stability in pseudoexfoliation syndrome. Acta Ophthalmol Scand 1999 Aug; 77(4): 406-9.

[9]. Georgios K, Christos G et al. Central corneal thickness in subjects with glaucoma and in normal individuals (with or without pseudoexfoliation syndrome). Clinical Ophthalmology. 2009; 1: 537-542. 